\title{
A Survey of Snakebite Management Knowledge Amongst Select Physicians in Hong Kong and the Implications for Snakebite Training
}

Hin T. J. Fung, FHKAM; Shing K. T. Lam, MRCSEd; Ka K. Lam, FHKAM; Chak W. Kam, FHKAM; Ian D. Simpson, BSc, PG (Dip)

From Emergency Medicine, Tuen Mun Hospital, Tuen Mun, New Territories, Hong Kong, People's Republic of China (Drs Fung, Lam, Lam, Kam); and Pakistan Medical Research Council, Islamabad, Pakistan, and KFBG China Programme, New Territories, Hong Kong, People's Republic of China (Dr Simpson).

\begin{abstract}
Objective.-The objective of the study was to assess the level of knowledge regarding snakebite management in doctors likely to treat such bites in the Special Administrative Region of Hong Kong in the People's Republic of China. Key concerns were doctor confidence, consistency of approach, use of anti-snake venom (ASV), and ancillary treatments. Hong Kong hospitals are equipped according to developed country standards, and knowledge therefore becomes the key factor in successful management.

Methods.-A predesigned questionnaire consisting of 29 multiple-choice questions was submitted to physicians likely to treat snakebite victims at all Hong Kong hospitals receiving such patients.

Results.-The key finding identified that only $29 \%$ of responding doctors were confident about treating snakebites. In the case of ASV selection between the 2 products available that deal with different species, $66 \%$ of doctors either were unsure of which to use or believed the 2 ASVs to be the same. The use of inappropriate clinical endpoints for ASV therapy suggests it is being used unnecessarily.

Conclusions.-There is clear room for improvement in the knowledge base and confidence level of physicians treating snakebites in Hong Kong. Key components of management, such as ASV choice, indications, dosing, and clinical endpoints for administration, were sources of confusion to the participants in this study. The results demonstrate the need for a locally developed and widely distributed snakebite management protocol.
\end{abstract}

Key words: snake bites, anti-snake venom, medical training, envenoming, World Health Organization, Hong Kong

\section{Introduction}

Recent focus within snakebite management has remained on the provision of anti-snake venom (ASV), with a particular focus on anti-venom quality. ${ }^{1}$ Considerable time and resources have been focused in regional meetings to produce even more stringent quality guidelines for a product that is described as unavailable. Despite medical education relating to snakebite management being described as an issue for nearly 30 years, very few initiatives have addressed it. ${ }^{2-5}$ Analysis of medical education and doctors' ability to effectively treat

Corresponding author: Ian D. Simpson, Kooran House, Nayathode, Kerala 683572, India (e-mail: iandsimpson@gmail.com). snakebite has established that traditional approaches utilizing Western textbooks and protocols developed with minimal local involvement are inadequate in India and Pakistan, areas with limited medical resources. ${ }^{6-9}$ The objective of this study was to assess doctors' knowledge of snakebite management in a developing country with comparable equipment to developed world counterparts.

The most important medically significant snakes in Hong Kong are the white-lipped pit viper (Cryptelytrops albolabris), the Chinese cobra (Naja atra), and the Chinese krait (Bungarus multicinctus), with additional venomous species, including the king cobra (Ophiophagus hannah), the banded krait (Bungarus fasciatus), the 
Table 1. Results of doctors' confidence in managing snakebite, experience of snakebite management, and primary source of treatment knowledge

\begin{tabular}{lc}
\hline Category & $\begin{array}{c}\text { Percentage (no.) } \\
\text { of responses }\end{array}$ \\
\hline $\begin{array}{l}\text { Doctors confident about treating snakebite } \\
\text { Doctors who have treated snakebite }\end{array}$ & $29(12)$ \\
$\begin{array}{l}\text { Doctors who have administered anti-snake } \\
\text { venom }\end{array}$ & $33(14)$ \\
$\begin{array}{l}\text { Doctors' source of knowledge: scattered, } \\
\text { local, overseas }\end{array}$ & $48(20)$ \\
\hline
\end{tabular}

red-necked keelback (Rhabdophis subminiatus helleri), the mountain pit viper (Ovophis monticola), and the point-scaled pit viper (Protobothrops muscrosquamatus $).{ }^{10-12}$

In 2007 there were 83 cases of snakebite recorded by the Clinical Data Analysis and Reporting System for Hong Kong (unpublished data). Hong Kong has no indigenous production of ASV and utilizes 2 main suppliers: Shanghai Institute of Biological Products (SIBP) and Thai Red Cross, Queen Saovabha Memorial Institute (TRC).

\section{Methods}

The Special Administrative Region of Hong Kong in the Peoples Republic of China is segmented into 7 public hospital clusters (Hong Kong East, Hong Kong West, Kowloon Central, Kowloon East, Kowloon West, New Territories East, and New Territories West), each with a main cluster hospital supported by a number of acute hospitals, 9 in total. Both cluster and acute hospitals are equipped with an accident and emergency department and are the mainstay of snakebite treatment.

A predesigned questionnaire (available at http://www. wem.org) was sent by email to officials at the 7 cluster and 9 acute hospitals in Hong Kong, with a request that they specifically ask doctors who treat or are interested in snakebite management to complete the questionnaire. This targeted method was adopted because it was regarded as the best method of ensuring that doctors with actual experience or interest were selected and in hopes of its providing a better representation of actual skill levels than would a blanket survey. A blanket approach attempting to survey all doctors in Hong Kong would have been fruitless, because many nonrelevant disciplines and doctors who do not treat snakebite would have been included. Although Hong Kong has more than 1500 doctors in its hospitals, only approximately 80 snake bites are reported each year. The questionnaire consisted of 29 multiple choice questions requiring either single or multiple answers based on the respondent's estimate of the correct answer.

Key areas of interest were included in the questionnaire: 1) origins of doctor's knowledge of how to treat snakebite, 2) doctor confidence in treating snakebites, 3) syndromic recognition among the medically significant species, 4) ASV administration criteria and clinical endpoints of ASV therapy.

\section{Results}

Responses to the survey were received from 42 doctors representing all 7 clusters, matching the distribution of snakebite in the SAR and thus achieving what we regard as a good sample, particularly because $52 \%$ had actually treated snakebites. Responses from each cluster and number of bites treated by that cluster in 2007 (as shown by the Clinical Data Analysis and Reporting System) were Hong Kong East: 5 responses/7 bites; Hong Kong West: 2/1; Kowloon Central: 3/6; Kowloon East: 5/2; Kowloon West: 5/9; New Territories East: 10/23; and New Territories West: 10/35. Two did not specify a cluster. Of the 16 total hospitals that treat snakebite, $63 \%$ responded specifically, including hospital name. The remainder specified only cluster and not hospital. Of the respondents, $65 \%$ were practicing in accident and emergency or in internal medicine. The remaining doctors worked in intensive care units, family medicine, orthopedics, and anaesthesiology.

Results of doctors' confidence in managing snakebite, experience of snakebite management, and primary source of treatment knowledge are presented in Table 1. Responding doctors demonstrated a low level of confidence in their ability to treat snakebite, with only $29 \%$ feeling confident and $\sim 50 \%$ of doctors who had treated snakebite not feeling confident.

The responding doctors' assessment of venom action of the key medically significant snake species in Hong Kong is found in Table 2.

In the case of krait bite, $50 \%$ (21) of doctors chose not correctly identifying the biting species early as the most significant barrier to effective treatment, and 31\% (13) identified the patient's arriving late as the most important factor. The Chinese cobra was regarded as presenting the highest mortality risk by $31 \%$ (13) of respondents, with $14 \%$ (6) unsure which species poses the greatest threat. In cases of white-lipped pit viper ( $C$ albolabris) bites with significant swelling, $31 \%$ (13) of respondents believed that there was a $<50 \%$ probability of developing compartment syndrome.

The physicians' choices of ASVs depending on findings of envenoming (syndromic recognition) are 
Table 2. Venom action selected by respondents for each of the key medically significant species

\begin{tabular}{lcccc}
\hline \multicolumn{1}{c}{ Species } & Cytotoxic & Hemotoxic & Neurotoxic & Myotoxic \\
\hline Naja atra & $29(12)^{*}$ & $26(11)$ & $57(24)$ & $19(8)$ \\
Bungarus multicinctus & $7(3)$ & $19(8)$ & $62(26)$ & $5(2)$ \\
Cryptelytrops albolabris & $41(17)$ & $52(22)$ & $19(8)$ & $21(9)$ \\
\hline
\end{tabular}

*The first number listed represents the percentage of responses and the number in parentheses the number of responses.

presented in Table 3. Regarding ASV initial dosage, of the 24 doctors who responded with an initial dose in the case of severe swelling involving the whole bitten limb, $38 \%$ (9) of respondents were not sure if they would administer SIBP or TRC ASV, 33\% (8) would administer TRC ASV, 17\% (4) believed both SIBP and TRC ASVs were the same, and $13 \%$ (3) would administer SIBP ASV. The ASV administration route of intravenous injection or continuous intravenous infusion was selected by $88 \%$ (37) of respondents and intramuscular administration by $12 \%$ (5). Initial ASV dosages in response to clinical signs and symptoms are found in Table 4.

In the case of timeframe to reassess whether a hemotoxically envenomed patient requires a second dose of ASV, 24\% (10) of respondents would wait 1 to 2 hours, $35 \%$ (15) would wait 3 to 4 hours, $14 \%$ (6) would wait 5 to 6 hours, and 26\% (11) did not know the period of time they would wait. For neurotoxic patients, $55 \%$ (23) would wait 1 to 2 hours, $16 \%$ (7) would reassess after 3 to 6 hours, and $26 \%$ did not know how long they would wait.

In the case of anaphylactoid reactions to ASV, $17 \%$ (7) of doctors believed they were due to snake venominduced anaphylactoid reactions, 59\% (25) attributed them to ASV-induced anaphylactoid reactions, and $14 \%$ (6) did not know the cause. The first line of treatment was identified as hydrocortisone and antihistamine by $57 \%$ (24), with $36 \%$ (15) of respondents preferring adrenaline. If adrenaline was employed in dealing with anaphylactoid reactions, 54\% (21) of respondents would utilize the subcutaneous route of administration, and $14 \%$ (6) would use intravenous administration.

Respondents' criteria for administering ASV and clinical endpoints for ASV administration are summarized in Table 5. In the case of neurotoxic bites, 50\% (12) of doctors who answered the question would administer at a maximum 20 vials of ASV to a patient with a confirmed krait bite and respiratory failure, and $43 \%$ (18) of respondents did not know how many vials they would use as an endpoint. In the case of neostigmine, 31\% (13) of doctors would deploy the drug in the case of Chinese cobra (26\% [11] vs the banded krait) and $21 \%$ (9) in the case of a bite from a Chinese krait. In the case of neostigmine dosage, $36 \%$ (15) of respondents did not know the dose they would employ.

In the case of recommended first aid treatment in the event of snakebite, $48 \%$ (20) of respondents opted for immobilization and transport as the only method, $17 \%$ (7) opted for tourniquet alone, and 14\% (6) chose pressure immobilization technique alone. Bite marks with 2 puncture wounds were regarded as inconclusive evidence as to whether the biting species was venomous or nonvenomous by $79 \%$ (33) of respondents; $12 \%$ (5) did not know.

\section{Discussion}

\section{ORIGIN OF DOCTOR KNOWLEDGE AND CONFIDENCE}

Doctor confidence is a key aspect of snakebite management in developing countries. Doctors who lack confidence will often withhold ASV and refer patients to other hospitals for treatment, thus increasing the time available to venom to bind to target tissues. ${ }^{13}$ Intuitively, doctor confidence in Hong Kong would be expected to be high; Hong Kong hospitals are highly equipped with modern equipment, such as ventilators, diagnostic testing, and modern accident and emergency departments. Problems faced by doctors in developing

Table 3. Physicians' choices of anti-snake venom (ASV), depending on symptoms of envenoming

\begin{tabular}{llllll}
\hline \multicolumn{1}{c}{ Symptom } & SIBP* & TRC* & Both ASVs are the same & Not sure & Do not know \\
\hline Ptosis & $26(11) \dagger$ & $17(7)$ & $12(5)$ & $26(11)$ & $19(8)$ \\
Swelling involving whole limb & $10(4)$ & $24(10)$ & $14(6)$ & $33(14)$ & $19(8)$ \\
\hline
\end{tabular}

*SIBP indicates Shanghai Institute of Biological Products; TRC, Thai Red Cross, Queen Saovabha Memorial Institute.

$\dagger$ The first number listed represents the percentage of responses and the number in parentheses the number of responses. 
Table 4. Initial anti-snake venom (ASV) dosage in response to clinical signs and symptoms

\begin{tabular}{|c|c|c|c|}
\hline Signs or symptoms & $\begin{array}{c}\text { Respondents who would } \\
\text { administer ASV }\end{array}$ & Do not know & $\begin{array}{c}\text { Initial mean } \\
\text { ASV vials }\end{array}$ \\
\hline Patient with incoagulable blood & $62(26) *$ & $38(16)$ & 3.3 \\
\hline Patient with ptosis & $57(24)$ & $43(18)$ & 4.0 \\
\hline Patient with swelling involving the whole bitten limb & $57(24)$ & $43(18)$ & 4.0 \\
\hline
\end{tabular}

*The first number listed represents the percentage of responses and the number in parentheses the number of responses.

countries, such as poor facilities at first point of contact with the victim, are thus not present. ${ }^{13}$ The low level of confidence evidenced $(29 \%)$ thus points towards the origin of knowledge as the main cause, particularly because nearly $50 \%$ of doctors who treated snakebites were not confident about having done so. The key textbook of medical education, previously identified as the primary source in another study, has been abandoned as the primary resource for snakebite due to the lack of local applicability. 7,9 "Scattered sources of knowledge both local and overseas," highlighted by $48 \%$ of doctors as the origin of their knowledge, is a clear indication of the lack of an adequate single, locally relevant training source that is widely distributed. ${ }^{6,12}$

\section{SYMPTOM RECOGNITION AMONG THE MEDICALLY SIGNIFICANT SPECIES}

Symptom recognition among the medically significant species also reinforces the need for relevant local protocols. The high level of attribution of neurotoxic symptoms to $N$ atra is likely due to the view that cobras are neurotoxic. However, many cobras are cytotoxic in effect, such as Naja nigricollis and Naja mossambica in Africa. ${ }^{2}$ Interestingly $N$ atra's reputation for spiting

Table 5. Criteria for administering anti-snake venom (ASV) and clinical endpoints for ASV administration

\begin{tabular}{lc}
\hline \multicolumn{1}{c}{ Symptom or circumstance } & $\begin{array}{c}\text { Percentage (no.) } \\
\text { of responses }\end{array}$ \\
\hline Significant swelling after Chinese cobra bite & $43(18)$ \\
Significant swelling after green pit viper bite & $51(21)$ \\
Leukocytosis & $2(1)$ \\
Any thrombocytopenia & $17(7)$ \\
Visible neurologic signs & $64(27)$ \\
Confirmed krait bite & $48(20)$ \\
ASV endpoint: when all symptoms reverse & $36(15)$ \\
ASV endpoint: when neurologic signs & \\
$\quad$ reverse & $36(15)$ \\
ASV endpoint: when blood is coagulable & $26(11)$ \\
ASV endpoint: when swelling subsides & $19(8)$ \\
ASV endpoint: do not know & $17(7)$ \\
\hline
\end{tabular}

venom may need revisiting, because experience capturing many hundreds of this species by a local specialist agency has not established a single case of venom spitting (personal communication Paul Crow, Kadoorie Farm \& Botanic Garden). The low recognition that $N$ atra causes almost exclusively cytotoxic symptoms, rather than systemic, may indicate that victims who require ASV in response to local swelling are facing a delay as doctors are waiting for neurotoxicity to develop. The low amount of realization that $B$ multicinctus is neurotoxic in nature is troubling, because all the krait species are neurotoxic, compounded by the dichotomy between the 2 krait species and by which ASV to administer. Only $52 \%$ of respondents identified $C$ albolabris as exhibiting hemotoxic symptoms, reflecting the preponderance of cytotoxic symptoms and the relatively low level of systemic symptoms. ${ }^{14}$

\section{ANTI-SNAKE VENOM ADMINISTRATION CRITERIA, DOSAGE, AND CLINICAL ENDPOINTS OF ANTI-SNAKE VENOM THERAPY}

In the case of the 2 most frequent biting species, the primary indication of envenomation is severe swelling, and thus, not unsurprisingly, significant swelling after both $C$ albolabris and $N$ atra bites was determined as grounds to administer ASV by $41 \%$ to $51 \%$ of the respondents. The use of ASV purely in the case of swelling without systemic symptoms is controversial, and there is no proven benefit to administration, making clear guidelines important. ${ }^{15,16}$ The appearance of coagulopathy in cases of $C$ albolabris is uncommon but a key systemic sign of envenomation. ${ }^{14,17-20}$

The fact that nearly half of all respondents regard a confirmed krait bite alone as being grounds for administering ASV is significant (Table 5). Presynaptic envenomation is almost universally accepted as not being "reversible."16,21-23 The destruction of synaptic vesicles can only be overcome by regeneration of the vesicles and the subsequent resumption of acetylcholine production, which is usually evident by 5 days after envenomation. ${ }^{21}$ The role of ASV is therefore to minimize the impact of the venom on the vesicles by neutralizing as much 
venom as possible before it can inflict damage. Antisnake venom is not curative in this scenario. Many textbooks, however, recommend that ASV be administered without any systemic symptoms because of the speed of onset of neurotoxic symptoms. ${ }^{7,24,25}$ This advice results in a waste of ASV, because the patient may well not be envenomed. Furthermore, there is no evidence that early administration of ASV results in better outcomes in neurotoxic bites. With the addition of excellent respiratory support equipment in Hong Kong hospitals, this advice is not only unproven but also unnecessary.

In the case of ASV dosage, the mean initial level administered in the case of all symptoms is low, reflecting the lack of guidance, even within the government-produced treatment advice. ${ }^{12}$ The high level of respondents who did not know the initial dose amount is further evidence of the need for a local protocol.

In the case of $C$ albolabris, the recommended dose of ASV in cases of coagulopathy is 4 to 10 vials of TRC ASV with the recognition that more doses may be required to restore coagulation. ${ }^{19,26,27}$ Guidelines from the Hong Kong Poisons Information Center, a specialist resource available for consultation by Hong Kong hospitals on toxicology issues, do not include guidelines for TRC ASV but do recommend 1 to 4 vials of SIBP Agkistrodon halys ASV that is of unproven effectiveness. ${ }^{10,14,28}$ In the case of $N$ atra, the recommended dose from the manufacturer is 2 vials of SIBP ASV, and the HPIC guidelines are 1 to 2 vials. ${ }^{27,28}$ The initial dose for $B$ multicinctus (ie, for symptoms of ptosis) is 5 vials of SIBP monovalent ASV, with local guidelines specifying 1 to 4 vials. $^{27,28}$ It is clear that research is needed to empirically establish the optimal initial volume and circulate this as part of the local protocol.

Clinical endpoints are varied and inadequate. The use of the endpoint "until all symptoms reverse" is perilous, because many symptoms will remain after circulating venom has been neutralized and the role of ASV is finished. This endpoint will necessitate ASV administration in the case of continuing swelling, respiratory failure, or necrosis if these are regarded as symptoms. Lack of clarity in the training material throughout snakebite literature as to the role of ASV in neutralizing free-flowing venom is a contributory factor. Useful clinical endpoints are restoration of coagulation, improvement in neurologic signs and symptoms, and as a final criterion the administration of sufficient ASV to neutralize the maximum amount of venom potentially administered by the species if known. ${ }^{4,9,13}$

Current recommendations are that hemotoxically envenomed patients are reevaluated after 6 hours and neurotoxically envenomed patients after 1 to 2 hours, because in the former this represents the period required for the liver to restore clotting factors and in the latter the faster onset of life-threatening symptoms requires more urgent review. ${ }^{4,6,9}$ These criteria are important to ensure that patients receive ASV in a timely manner and that administering repeat doses too early does not waste ASV.

\section{ANTI-SNAKE VENOM SELECTION BY THE TREATING PHYSICIAN}

The selection of ASV is a key concern in Hong Kong, because a number of ASVs are available to doctors from 2 manufacturers: SIBP and TRC. SIBP supplies 3 ASVs: 1) monovalent Naja naja (atra) antivenin ( $N$ atra), 2) monovalent $B$ multicinctus antivenin (B multicinctus), 3 ) monovalent $A$ halys antivenin (A halys). TRC supplies 2 ASVs: 1) monovalent banded krait antivenin ( $B$ fasciatus), 2) monovalent green pit viper antivenin (Trimeresurus albolabris).

In the case of the 2 most frequent biting species, $C$ albolabris and $N$ atra, the most common sign of envenoming is significant local swelling. $N$ atra is a cytotoxic and not neurotoxic cobra. ${ }^{14,18-20}$ The choice of ASV between TRC green pit viper antivenin and SIBP monovalent $N$ naja (atra) antivenin is thus problematic in cases of victims bitten by unidentified snakes and presenting with local swelling, and this is reflected in the respondents' choices. There is a view, supported in the literature, that SIBP ASV does not effectively neutralize the venom of $C$ albolabris. ${ }^{14,29}$ In the case of neurotoxic species $B$ multicinctus and $B$ fasciatus, visible neurologic signs do not facilitate the correct choice between the different krait ASVs made by SIBP and TRC, because both SIBP may be applicable, as in the case of $B$ multicinctus or TRC in B fasciatus.

The level of respondents who either did not know or were not sure which ASV to administer was between $45 \%$ and $52 \%$, which is problematic. Hong Kong's recent absorption into the People's Republic of China has resulted in ASV being supplied to Hong Kong by the Chinese indigenous supplier. However, $C$ albolabris and $B$ fasciatus are not included within the venom mix used for any of the SIBP ASVs. Given this exposure and the level of confusion as to which ASV to select in response to symptoms, it may be useful to reassess the current ASV products for suitability and ease of administration across China and design a new, broader range polyvalent ASV.

\section{METHODS OF TREATING ADVERSE REACTIONS TO ANTI-SNAKE VENOM}

Similar to previous findings, ${ }^{9} 57 \%$ of respondents selected hydrocortisone and antihistamine as the preferred treatment of anaphylactoid reactions, despite 
intramuscular adrenaline being the recommended approach due to its speed of action. ${ }^{9,30,31}$ Also as found previously, ${ }^{9}$ respondents who selected a route for administration of adrenaline most commonly chose subcutaneous. The approach to dealing with adverse reactions to ASV needs clear guidance, because simply managing the adverse reaction does not constitute success. Anti-snake venom is discontinued, and thus more venom is permitted to bind to target cells. In snakebite management, success constitutes the most rapid resolution of the reaction and recommencing ASV therapy. Intramuscular adrenaline, with its faster action and therefore likely reduced reaction period, is essential in snakebite treatment, because the victim requires ASV to neutralize the venom. It is interesting that despite Hong Kong hospitals' being equipped with excellent emergency department equipment, where recommendations have suggested that intravenous adrenaline can be used, few respondents selected this method. ${ }^{29,30,32}$

\section{OTHER KNOWLEDGE ISSUES}

In common with previous reports, first aid methods remain less than optimal, with reliance on tourniquets or their equivalent that attempt to squeeze the venom from the wound, cutting, and suction. ${ }^{9,10}$ Compartment syndrome is still regarded by many doctors in Hong Kong as a common risk in severe swelling, although the evidence is scant that the actual intracompartmental pressure reaches levels that justify fasciotomy. ${ }^{6}$ This represents an area in which prospective work could be carried out using objective measures of intracompartmental pressure.

Limitations of this study include its small sample size and the fact that there was no way for us to determine a response rate to our questionnaire, because we could not know the number of physicians to whom each hospital forwarded the email request for participation. Furthermore, it is impossible to know that those who distributed the questionnaire at each hospital sent it to all physicians we hoped to have targeted. Hong Kong is, however, a small special administrative region of China that is a relatively self-contained area, with only 16 hospitals that treat snakebite. Given that the response rate from institutions was at least $63 \%$ in terms of named treating hospitals and the majority of responses were from the areas with the highest incidence of snakebite, we believe the results accurately represent the current knowledge and confidence levels of physicians interested in treating snakebites in Hong Kong.

\section{Conclusions}

Although medical education has been frequently identified as a priority, very little work has been carried out to improve the situation. ${ }^{1-5}$ Recent work has enabled a specific analysis of this problem, which has been long overdue and is echoed in this study. ${ }^{9}$ What is now required is a concerted effort by such organizations as the World Health Organization to address the problem by providing relevant locally developed protocols, as an alternative to concentrating on attempting to improve the quality of a product they themselves admit is unavailable. $^{1,33}$ Unless currently produced ASV is used effectively, it makes little sense to speak of shortages. Additional ASV will provide few benefits unless it is used effectively and all aspects of snakebite treatment are improved.

\section{References}

1. World Health Organization. Rabies and Envenomings: A Neglected Public Health Issue. Geneva, Switzerland: World Health Organization; 2007.

2. World Health Organization. Progress in the characterization of venoms and standardization of antivenoms. W.H.O. Offset Publ. 1981;58:1-44.

3. Theakston RDG, Warrell DA, Griffiths. Report of a W.H.O. workshop on the standardization and control of antivenoms. Toxicon. 2003;41:541-557.

4. Government of India. National Snakebite Protocol. New Delhi, India: Health \& Family Welfare Department; 2007.

5. Simpson ID, Quraishi NA, Qureshi HI. Snakebite Management in Pakistan: A Guide to the Latest Methods of Treatment. Islamabad: Pakistan Medical Research Council; 2008.

6. Warrell DA. W.H.O./SEARO Guidelines for the clinical management of snakebite in the Southeast Asian Region. SE Asian J Trop Med Pub Health. 1999;1:1-85.

7. Auerbach PS, Norris RL. Disorders caused by reptile bites and marine animal exposures. In: Braunwald E, Fauchi AS, Kasper DL, et al, eds. Harrison's Principles of Internal Medicine. 16th ed. New York, NY: McGraw-Hill; 2004:2593-2600.

8. Rawlins M, Vale JA. Drug therapy and poisoning. In: Kumar P, Clark M, eds. Clinical Medicine. 6th ed. Philadelphia, PA: Elsevier; 2005:991-1024.

9. Simpson ID. A study of the current knowledge base in treating snakebite amongst doctors in the high risk countries of India and Pakistan: does snakebite treatment training reflect the local requirement? Trans Roy Soc Trop Med Hyg. 2008;102:1108-1114.

10. Ng W, Cheung WL. Snake bites in Hong Kong: a prospective study on epidemiology and pre-hospital management. HKJEM. 1997;4:68-73.

11. Karsen SJ, Lau MW, Bogadek A. Hong Kong Amphibians and Reptiles. Hong Kong SAZ, China: Provisional Urban Council, Hong Kong; 1998.

12. Chan KFS, Cheung KS, Ho CY, Lam FN, Tang WS. A Field Guide to the Venomous Land Snakes of Hong Kong. Hong Kong SAZ, China: Friends of the Country Parks, Cosmos Books Limited; 2006. 
13. Simpson ID. Snakebite management in India, the first few hours: a guide for primary care physicians. J Indian Med Assoc. 2007;105:324-335.

14. Fung HT, Kam CW. Efficacy and safety of snake antivenom therapy: experience of a regional hospital. Hong Kong J Emerg Med. 2006;13:70-78.

15. Rojnuckarin P, Chanthawibun W, Noiphrom J, Pakmanee $\mathrm{N}$, Intragumtornchai T. A randomised, double-blind, placebo-controlled trial of antivenom for local effects of green pit viper bites. Trans Rou Soc Trop Med. 2006;100: 879-884.

16. Gutiérrez JM, Lomonte B, León G, Rucavado A, Chaves F, Angulo Y. Trends in snakebite envenomation therapy: scientific, technological and public health considerations. Curr Pharm Des. 2007;13:2935-2950.

17. Mahasandana S, Rungruxsirivorn Y, Chantarangkul V. Clinical manifestations of bleeding following Russell's viper and Green pit viper bites in adults. Southeast Asian J Trop Med Public Health. 1980;11:285-293.

18. Cockram CS, Chan JCN, Chow KY. Bites by the whitelipped pitviper (Trimeresurus albolabris) and other species in Hong Kong: a survey of 4 years experience at the Prince of Wales Hospital. J Trop Med Hyg. 1990;93:79-86.

19. Hutton RA, Looareesuwan S, Ho M, et al. Arboreal green pit vipers (genus Trimeresurus) of south-east Asia: bites by T. albolabris and T. macrops in Thailand and a review of the literature. Trans Roy Soc Trop Med Hyg. 1990;84:886874.

20. Chan TYK, Critchley JAJH. An epidemiological study of the snake bites in the New Territories East, Hong Kong. Ann Trop Med Para. 1994;88:219-221.

21. Dixon RW, Harris JB. Nerve terminal damage by Bbungarotoxin. Am J Pathol. 1999;154:447-455.

22. Harris JB, Goonetilleke A. Animal poisons and the nervous system: what the neurologist needs to know. J Neurol Neurosurg Psychiatr. 2004;75:40-46.
23. Cheng AC, Currie BJ. Venomous snakebites worldwide with a focus on the Australia-Pacific Region: current management and controversies. I Intensive Care Med. 2004;19:259-269.

24. Bambery P, Malhotra HS, Chugh KS. Clinical problems of snakebites in India. In: Tu A, ed. Toxin Related Diseases. New Delhi, India: IBH; 1993:419-443.

25. Chippaux JP. Snake Venoms and Envenomations. Malabar, FL: Krieger Publishing Company; 2006:211-246.

26. Mitrakul C. Clinical features of viper bites in 72 Thai children. Southeast Asian J Trop Med Public Health. 1982; 13:628-636.

27. Warrell DA. Bites by venomous snakes outside the Americas. In: Auerbach PS, ed. Wilderness Medicine. St. Louis, MO: Mosby; 2007:1086-1123.

28. Hong Kong Poisons Information Centre. Mechanism for the Emergency Supply of Antidotes in HA Hospitals. Hong Kong, China: Hong Kong Poisons Information Centre; 2008.

29. Yang JYK, Hui H, Lee ACW. Severe coagulopathy associated with white-lipped green pit viper bite. Hong Kong Med J. 2007;13:392-395.

30. Lieberman P. Use of epinephrine in the treatment of anaphylaxis. Curr Opin Allergy Clin Immunol. 2003;3: 313-318.

31. McLean-Tooke APC, Bethune CA, Fay AC, Spickett GP. Adrenaline in the treatment of anaphylaxis: what is the evidence? BMJ. 2003;327:1332-1335.

32. Sampson HA, Mendelson L, Rosen JP. Fatal and near-fatal anaphylactic reactions to food in children and adolescents. N Engl J Med. 1992;327:380-384.

33. Ghosh S, Maisnam I, Murmu BK, Mitra PK, Roy A, Simpson ID. A locally developed snakebite management protocol significantly reduces overall anti snake venom utilization in West Bengal, India. Wilderness Environ Med. 2008;19:267-274. 


\section{Snakebite Management Questionnaire}

Questions are directed towards snakebite scenarios in Hong Kong. No question is compulsory. Please circle or write down the answer which may be more than one. ASV = Anti-snake venom

1. From where did you learn how to treat snakebite?
a) Harrison's Principles of Internal Medicine
b) Tintinalli, Emergency Medicine
c) College guidelines
d) Own departmental guidelines
e) From experienced doctors, as I went along
f) From scattered sources, local \& overseas

2. Do you feel confident to treat snakebite now?
a) Yes
b) No

3. Have you treated a snakebite case?
a) Yes
b) No

4. Have you given ASV?
a) Yes
b) No

5. The common complications of Chinese cobra bites:
a) Cytotoxic
b) Hemotoxic
c) Neurotoxic
d) Myotoxic

6. The common complications of krait bites:
a) Cytotoxic
b) Hemotoxic
c) Neurotoxic
d) Myotoxic

7. The common complications of Green pit viper bites:
a) Cytotoxic
b) Hemotoxic
c) Neurotoxic
d) Myotoxic

8. Among all venomous snakebite, cobra bites:
a) Seldom cause respiratory failure
b) Are the rarest
c) Are the commonest
d) Result in the highest mortality

9. Does a bite-mark with 2 puncture holes indicate?
a) Non-venomous bite
b) Venomous bite
c) Inconclusive

10. Swelling after Green pit viper bite generally develops within:
a) 1 hour
b) 2 hours
c) 4 hours
d) 8 hours

11. If patients present with significant swelling after suspected Green pit viper bite,
a) Green pit viper bite is in doubt
b) $<50 \%$ chance of compartment syndrome
c) Paresthesia is associated
d) Fever usually accompanies

12. For krait bite, which one hinders effective treatment most?
a) Getting the $1^{\text {st }}$ ASV dose correct
b) Anaphylaxis to ASV
c) Patient arriving late
d) Not correctly identifying the snake-species at beginning

13. Which first-aid method would you recommend for snakebite?
a) Tourniquet
b) Pressure immobilisation
c) Suction
d) Immobilisation \& transport

14. How should ASV be administered?
a) IM
b) IV injection
c) Continuous IV infusion
d) Locally at bite-site 
15. Which of the following are criteria for giving ASV?

a) Significant swelling after Chinese cobra bite b) Significant swelling after Green pit viper bite

c) Leukocytosis d) Any degree of thrombocytopenia e) visible neurological signs f) Confirmed krait bite

16. If victim had incoagulable blood, how many vials of ASV would you give in the first dose?

17. If victim had ptosis, how many vials of ASV would you give in the first dose?

.........vials

18. If victim had severe swelling involving the whole limb, how many vials of ASV would you give in the first dose? vials

19. If the patient had ptosis, your first choice of ASV would be that manufactured in:
a) Shanghai
b) Thailand
c) a $\&$ b are the same
d) Not sure

20. If the patient had severe swelling involving the whole limb, your first choice of ASV would be that manufactured in:
a) Shanghai
b) Thailand
c) a \& b are the same
d) Not sure

21. Skin rash after giving ASV is usually due to:
a) Snake venom anaphylaxis
b) Snake venom anaphylactoid reaction
c) ASV anaphylaxis
d) ASV anaphylactoid reaction

22. Which drug is the first to be used to treat an ASV reaction?
a) Hydrocortisone
b) Anti-histamine
c) Hydrocortisone + Anti-histamine
d) Adrenaline

23. If you administered ASV to a haemotoxic patient, how many hours would you wait to determine if more ASV was necessary?
a) $1-2 \mathrm{hrs}$
b) $3-4$ hrs
c) $5-6 \mathrm{hrs}$
d) $7-8$ hrs

24. If you administered ASV to a neurotoxic patient, how many hours would you wait to determine if more ASV was necessary?
a) $1-2 \mathrm{hrs}$
b) $3-4$ hrs
c) $5-6$ hrs
d) $7-8 \mathrm{hrs}$

25. If you had to administer adrenaline to a patient with urticaria from an ASV reaction, which method would you use?
a) Subcutaneous
b) IM
c) IV

26. When should you stop administering ASV to an envenomed patient?
a) When swelling subsides
b) When bleeding stops
d) When all symptoms reverse
e) When neurological signs reverse
c) When blood is coagulable

27. What is the maximum number of vials of ASV that you would give to a patient with confirmed krait bite and respiratory failure?
a) 5 Vials
b) 10 Vials
c) 15 Vials
d) 20 Vials
e) $20+$ Vials

28. What dose of Neostigmine would you administer to an adult patient with neurotoxic envenomation?
a) $10 \mathrm{mg}$ IV
b) $10 \mathrm{mg} \mathrm{IM}$
c) $1.5 \mathrm{mg} \mathrm{IV}$
d) $1.5 \mathrm{mg} \mathrm{IM}$

29. Agrainst which species would you use Neostigmine?
a) Banded krait
b) Chinese Cobra
c) Green pit viper
d) Chinese Krait

30. Your cluster / hospital

31. Your department a) Clinical:

b) Non-elinical;

32. Your E-mail if you want to receive the correct answers 